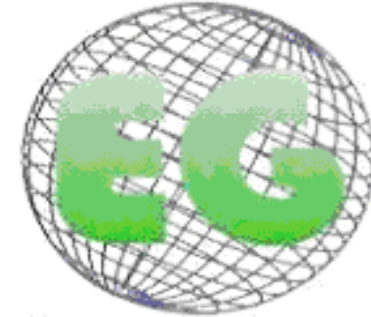

ISSN 1695-6141

$\mathrm{N}^{\circ} 21$

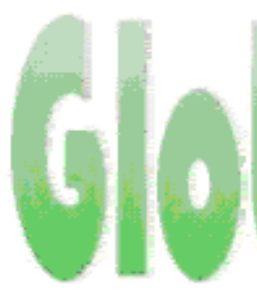

Revista electrónica trimestral de Enfermería

www.um.es/egloball

Enero 2011

DOCENCIA - INVESTIGACIÓN

\title{
EL PROCESO DE EVALUACIÓN DE LA FORMACIÓN EXTRACURRICULAR EN LAS UNIDADES DE SALUD DE RIO DE JANEIRO
}

O PROCESSO DE AVALIACÃO DO ESTÁGIO EXTRACURRICULAR EM SAÚdE NAS UNIDADES DE SAÚDE DO RIO DE JANEIRO

*Pereira da Silva, T., "*Lopes de Abreu da Fonseca, AP., ***Simeão dos Santos, MS.

\begin{abstract}
*Miembro del Núcleo de Pesquisa Educação e Saúde em Enfermagem (NUPESEnf ) **Enfermera. Miembro del Núcleo de Pesquisa Educação e Saúde em Enfermagem (NUPESEnf) ***Doctora en Enfermería. Profesora Adjunta del Departamento de Metodologia da Enfermagem da Escola de Enfermagem Anna Nery /UFRJ. Pesquisadora y Miembro del Núcleo de Pesquisa Educação e Saúde em Enfermagem (NUPESEnf). Brasil.
\end{abstract}

Palabras clave: Educación. Prácticas Clínicas. Cuidados de Salud.

Palavras chave: Educação. Estágio clínico. Assistência à Saúde

Keywords: Education. Clinical Clerkship. Delivery of Health Care.

\section{RESUMEN}

La investigación tiene su objetivo en la organización didáctica de la propuesta de formación, con foco en el planeamiento y evaluación de la Formación Extracurricular Remunerada (EER). El EER es una actividad no obligatoria que imprime en el estudiante el compromiso, destreza e independencia financiera. Objetivamos: identificar las modalidades de evaluación emprendidas y describir las condiciones operacionales de las Instituciones ofrecidas para la formación. Investigación cualitativa, descriptiva y exploratoria. Los escenarios fueron seis unidades de salud vinculadas a la Secretaría de Salud del Estado de Rio de Janeiro. Los sujetos fueron los diecinueve estudiantes de las áreas de Enfermería, Farmacia y Medicina, así como los presidentes de los Centros de Estudios de las unidades. Los métodos evaluativos utilizados fueron: estudios de casos, relatos de pesquisa, registro de asistencia prestada, participación en reuniones, trabajo de conclusión de formación y registro del libro de órdenes y ocurrencias. Las unidades de Salud deben disponer de recursos para componer el escenario ideal para la práctica y formación de los futuros profesionales

\section{RESUMO}

A pesquisa tem seu objeto na organização didática da proposta de estágio, com foco no planejamento e avaliação do Estágio Extracurricular Remunerado (EER). O EER é uma atividade não-obrigatória que imprime no estudante o compromisso, destreza e independência financeira. Objetivamos: identificar as modalidades de avaliação empreendidas e descrever as condições 
operacionais das Instituições oferecidas ao estagiário. Pesquisa qualitativa, descritiva e exploratória. Os cenários foram seis unidades de saúde vinculadas à Secretaria de Saúde do Estado do Rio de Janeiro. Os sujeitos foram os dezenove estudantes das áreas de Enfermagem, Farmácia e Medicina, bem como os presidentes dos Centros de estudos das unidades. Os métodos avaliativos utilizados foram: estudos de casos, relatórios de pesquisa, registro de assistência prestada, participação em reuniões, trabalho de conclusão de estágio e registro do livro de ordens e ocorrências. As unidades de Saúde devem dispor de recursos para compor o cenário ideal para a prática e formação dos futuros profissionais.

\section{ABSTRACT}

The research has its object the organization of the proposed didactic training, focused on planning and evaluation of extracurricular Paid Internship (EER). The EER is a non-compulsory activity which prints student in the commitment, skill and financial independence. Aim: to identify the methods of assessment undertaken and describe the operating conditions of the institutions offered to the trainee. Qualitative research, descriptive and exploratory. The six scenarios were linked to health units of the Secretariat of Health of the State of Rio de Janeiro. The subjects were nineteen students in the areas of Nursing, Pharmacy and Medicine, and the chairmen of the study centers and units. The evaluation methods were used: case studies, research reports, record of assistance, participation in meetings, job training and completion of the registration book of orders and events. The units of Health must have sufficient resources to make the ideal setting for the practice and training of future professionals.

\section{CONSIDERACIONES INICIALES}

Para contextualizar la evaluación de las etapas extracurriculares remuneradas (EER) es necesario conocer el momento en que ocurrió la institucionalización, la legislación vigente en la época y las políticas públicas que amparan esta modalidad pedagógica, englobando las estrategias de evaluación didáctica de los estudiantes de enseñanza superior en el área de la Salud por los supervisores de las Instituciones de Salud.

La investigación basa su objeto de estudio en la organización didáctica de la propuesta de etapa referente al plan de enseñanza y la evaluación del EER. A partir de aquí se objetiva: identificar las modalidades de evaluación emprendidas por la SESDEC/RJ para los EER y describir las condiciones operacionales de las Instituciones que se presentan como escenario de práctica a los estudiantes.

EI EER se constituye en parte del proceso didáctico-pedagógico que posibilita al educando contacto con una actividad real, para la adquisición de experiencia, ofreciendo al estudiante herramientas que compondrán su futuro carácter profesional, dándoles seguridad y postura crítica ante los enfrentamientos cotidianos de la profesión. Es una actividad no obligatoria y, de manera general, bien vista por las instituciones de Salud, por algunos docentes y estudiantes, imprimiendo el interés y compromiso por la profesión, además de proporcionar experiencia profesional y destreza manual.

Los estudiantes buscan los EER con el objetivo de obtener experiencia profesional, independencia financiera y habilidades necesarias para el ingreso en el mercado de trabajo. La búsqueda del EER objetiva la necesidad de perfeccionamiento, ya que las habilidades prácticas en el ejercicio de la profesión son trabajadas más efectivamente en los últimos períodos del Curso de Graduación. 
En este estudio, la adquisición de habilidades por los estudiantes de enfermería, coincide a nivel personal, con el recibimiento de la bolsa-auxilio; y profesional, con la obtención de destreza para asegurar las competencias profesionales, de conocimiento teórico acumulado y de certificado al término del estadio extracurricular. La necesidad de practicar deriva de los bolsistas al no conseguir realizar las actividades requeridas por el número elevado de estudiantes en curso de graduación, necesidad de estabilidad en el puesto de trabajo ( homo faber) y la busca por la "vida boa", con el apoyo de la bolsa de estudios"

Justificamos la importancia de este estudio para la comprensión de los aspectos evaluativos de los EER, la necesidad de operacionalización de ese sistema formador extramuros de la Universidad, permitir una reflexión y revisión de las políticas vigentes que, seguramente, reforzarían la importancia de los estadios extracurriculares en Salud en la formación profesional de dos estudiantes.

En la década de los sesenta habían estadios voluntarios remunerados para estudiantes de la Enseñanza Superior, vinculados al Servicio Público Federal (Proyecto Integración, Proyecto Rondon, Operación Mauá, Programa Asistencial "Bolsa de Trabajo", Programa Especial de Bolsas de Estudio y de Monitor, objetivando la participación en el proceso de integración nacional ${ }^{1}$. Muchos se convirtieron en funcionarios de grandes empresas después del período de estadio extracurricular ${ }^{2}$.

Estas etapas existieron en un momento político en que eran tomadas providencias para contención de gastos públicos en recursos humanos y materiales afectando, principalmente, a los hospitales.

La institucionalización de los estadios extracurriculares (EE) se dio en el Estado de la Guanabara, en 1967, cuando la ciudad atravesaba por una epidemia ocasionada por el gran volumen pluviométrico. Ese escenario se confrontaba con la situación deficitaria de la red de servicio, que se fue deteriorando a lo largo de los años. Faltaban equipamientos y el cuadro de servidores técnico administrativos, según el histórico de la Secretaría de Salud del Estado de la Guanabara- SUSEME, era deficiente en número, fallos, muchas veces, sin cualificación y con media de edad bastante elevada ${ }^{3}$.

Las propuestas de mejora de la calidad de la asistencia a la población trillaron dos vertientes. En una de ellas, habría un cambio estructural de las unidades y equipamientos y, la otra, cualificaría el cuadro de funcionarios para trabajar con la nueva propuesta de funcionamiento de las unidades de salud. Ambas tendían a la extinción de los vicios y de las tergiversaciones sufridas a través de años, así como los desvíos de funciones que contribuyen a la deficiencia del trabajo ${ }^{3}$. La propuesta pedagógica instituida a partir del régimen militar surgió en el sentido de operacionalizar el proceso educacional ${ }^{4}$, donde había la necesidad de dominar la técnica y el tiempo para ejecutarla.

Las dos propuestas no fueron acompañadas de cambios salariales, lo que provocó evasión del personal técnico para otras áreas de mejor remuneración, reduciendo, por lo tanto, la productividad de los servidores que permanecieron, ya que la demanda de trabajo no fue alterada frente a la nueva realidad.

La entrada de los estudiantes de Medicina y de profesionales afines en el servicio estadual fue asegurada por la proposición de las rectorías de las Universidades y las direcciones de las Facultades albergadas en el Estado de la Guanabara, posibilitando la integración de los hospitales estaduales a las Instituciones de EnseñanzaSuperior, con la pretensión de viabilizar una aprendizaje altamente cualificado por los servicios especializados y el hecho 
de que los hospitales estaban siendo preparados con instalaciones que dignificarían el ejercicio de las profesiones ${ }^{5}$.

El interés de la Universidad en dinamizar la formación y aprovechar las potencialidades de los alumnos favoreció el proyecto de extensión universitaria, que propicia la retro alimentación de los mecanismos de la enseñanza aprendizaje, de la práctica asistencial formal y orientación para los grandes problemas sociales ${ }^{6}$.

En 1967, el Ministerio del Trabajo instituyó la Portaría 1002/1967, que regía la relación empresa estudiante de la Enseñanza Superior y de la Enseñanza Media, a través del contrato padrón del estadio. Por esa ley, el contrato debe contener, obligatoriamente, el local, periodo y horario del estadio, proveer al estudiante de cobertura de seguro contra accidentes personales durante el período de estadio, ya que no tenía vínculo de empleo y la concesión del número de la cartera profesional del aprendiz ${ }^{7}$.

El EE era garantizado por la Ley 6 494-07/1977 la cual fue revocada y, actualmente, sustituida por la Ley 11 788, de 25 de septiembre de 2008 que trata del estadio de estudiantes efectivamente matriculados en establecimientos de Enseñanza Superior y Media regular o complementario, público o privado, para el entrenamiento práctico y perfeccionamiento técnico, cultural y científico en la línea de formación ${ }^{8}$.

El día 19 de Junio de 2008 fue publicada, en el Diario Oficial del Estado de Rio de Janeiro, la Resolución N. 315 de 30 de mayo de 2008 que aprueba el reglamento de estadios de nivel medio y superior en unidades prestadoras de servicios de la red propia de la Secretaría de Estado de Saúde e Defesa Civil (SESDEC/RJ) ${ }^{9}$.

La Ley trajo cambios en la organización de los estadios curriculares y extracurriculares de nivel superior, en lo que se refiere a: vacaciones remuneradas, contrato de etapa con término de compromiso del estudiante, empresa y universidad, límite de vacantes por campo, auxilio transporte y bolsa auxilio y la reducción de la carga horaria de trabajo a seis horas diarias.

El EE se caracteriza por la no obligatoriedad, sin embargo, para su efectivación debe constituir convenio entre la Institución de Salud y escolar

Sin embargo, esa modalidad de estadio es vista casi siempre de manera positiva por las instituciones de salud y escolar, por los estudiantes y por algunos docentes, ya que, por no ser obligatoria, demuestra el interés y el compromiso del estudiante por la profesión, además de proporcionar mayor experiencia profesional y destreza manual.

Profesionales de éxito poseen en su histórico, períodos de EE y gran parte de las empresas exige del contratado competencia profesional, lo que muchas veces no se obtiene solo con el $\mathrm{EE}^{2}$. El estudiante que, durante la selección para empleo, presenta la declaración de EE, demuestra su interés por la profesión, además de mayor habilidad y experiencia para funciones exigidas ${ }^{10}$.

Los estudiantes buscan los EE con el objetivo de adquirir experiencia profesional e independencia financiera y buscan en ese estadio, adquirir las habilidades exigidas por el mercado de trabajo ${ }^{5}$.

Reconociendo las situaciones en su campo práctico, el rendimiento teórico del estudiante que desarrolla actividades extracurriculares es mayor, ya que ahora, él tiene otra visión de la teoría y de la práctica porque la vivencia práctica le permitió identificar la teoría con su propia 
acción ${ }^{5}$. Además, por no poseer las características de estadio curricular, fragmentadas y de corta duración, el EE posibilita al estudiante la vivencia de los problemas profesionales en su escenario natural ${ }^{4}$.

Además, los estudiantes buscaban el EER también como fuente de remuneración y de complementación del aprendizaje práctico ${ }^{10}$. La bolsa auxilio representa para el estudiante una complementación de la renta familiar ${ }^{11}$.

Pero, si por un lado los EE benefician a los estudiantes, por otro, las Instituciones salen beneficiadas. Gran parte de las empresas utiliza el estadiario como mano de obra barata en I lugar de la contratación de funcionarios que, por ser profesionales ya formados, estarían mejor remunerados. La presencia de estudiantes en las Instituciones, trae consigo una constante actualización a los profesionales de salud, ya que estos son factores de reciclaje, aunque algunos profesionales no tengan esa visión crítica ${ }^{5}$.

El estadio debe ser transformado en instrumento de aprendizaje transformador, libertador y comprometido con la realidad y vuelta para ella, pues, el impacto de la transición de la vida académica para la profesional puede verse disminuida a través de la experiencia de trabajo sistematizado, crítico y participativo ${ }^{4}$.

Sin embargo, para que el estadio posea tales cualidades, debe poseer recursos técnicos para que el aprendizaje sea hecho de forma efectiva. Un campo bien preparado ofrece condiciones para que el estudiante aproveche bien la horas que le son ofrecidas para el estadio, permitiéndole desarrollar sus potencialidades ${ }^{12}$.

EI EE debe servir como complemento de la práctica vista en el estadio curricular, para que el estudiante llegue al mercado de trabajo confiado y seguro para desempeñar sus funciones. De este modo, mira a la relación de la teoría con la práctica y la formación del estudiante para la vida profesional.

Es necesaria la conquista de la autoconfianza para que el futuro profesional esté seguro de sus acciones y palabras. Es fundamental, por lo tanto, desarrollar el sentido de confianza, para que, la persona pueda adquirir la fuerza necesaria para el gobierno del propio comportamiento. Una persona que no tenga desarrollado adecuadamente el sentido de confianza, dudará en luchar por la autonomía, sentirá necesidad de dependencia y temerá lanzarse a una acción ${ }^{13}$.

\section{MATERIAL Y MÉTODO}

Se trata de una investigación cualitativa, descriptiva y exploratoria, ya que el abordaje cualitativo de un problema, además de ser una opción del investigador, se justifica, sobre todo, por ser una forma adecuada para entender la naturaleza de un fenómeno social ${ }^{14} \mathrm{y}$ seleccionado el tipo de estudios multicasos, por ser una categoría de pesquisa cuyo objeto es una unidad que se analiza profundamente ${ }^{15}$.

Los escenarios del estudio fueron las unidades de salud que ejecutan los EE vinculadas a la Secretaria de Saúde e Defesa Civil do Estado do Rio de Janeiro: HEMORIO, Hospital Estadual Albert Schweitzer (HEAS), Hospital Estadual Adão Pereira Nunes (HEAPN), Hospital Estadual Pedro II (HEPII), Hospital Estadual Getúlio Vargas (HEGV) y Hospital Estadual Rocha Faria (HERF).

Los sujetos del estudio fueron los estudiantes del área de Salud vinculados al EER de la SESDEC/RJ que se dispusieron a responder a los cuestionamientos, así como los 
presidentes de los Centros de estudio vinculados a las unidades de Salud del Estado de Rio de Janeiro.

La pesquisa fue aprobada por el Comité de Ética en Pesquisa de la Escola de Enfermagem Anna Nery-EEAN/Hospital Escola São Francisco de Assis-HESFA bajo el número 24/08.

Fueron realizados análisis documental y entrevista con los responsables de la ejecución del EE en las Unidades. El itinerario de entrevista semiestructurada contuvo cuestiones abiertas con quesitos disertativos, para que los sujetos de la pesquisa pudiesen expresarse de manera más espontánea. La técnica de entrevista semiestructurada fue empleada, donde las declaraciones con los sujetos de la pesquisa se destinaron a una mayor profundización sobre las actividades desarrolladas por los alumnos en el campo práctico.

Fue solicitado a las Instituciones de estudio y aprobación de la inserción del nombre y de que su representante legal pudiese proporcionar las informaciones relativas al estadio.

Los hallados recibieron tratamiento de transcripción literal, siendo registrados por entero, seguidos de aprobación y validación de los hallados por los comparecientes.

Después de la lectura y análisis temática, para retratar el posicionamiento de los sujetos, en el contexto en que fueron producidos, se construyeron categorías de análisis que presentan la organización didáctica de la propuesta de estadio referente al plan de la enseñanza y a la evaluación del estadio.

\section{RESULTADOS}

\section{1) PRESENTANDO A LOS ESTADIARIOS (LOS EN PRÁCTICAS) Y SUS ESCENÁRIOS}

El estadio extracurricular sigue las determinaciones de la Resolución N. 1460 de 11 de febrero de 2000 que estipula el estadio remunerado de nivel superior $Y$ de nivel medio, a través de concurso público, elaborado por la SESDEC. Otra modalidad de estadio extracurricular, regulado por la Resolución N. 1459 de 11 de febrero de 2000, es el estadio remunerado en emergencia, denominado académico bolsista, para estudiantes de nivel superior.

Este concurso es elaborado por la División de Formación, Posgrado y Residencia de la Superintendencia de Gestión Participativa y Educación en Salud de la Secretaría de Estado de Salud y Defensa Civil. Las vacantes en las unidades de salud serán distribuidas de acuerdo con la solicitación de las mismas por los respectivos Centros de Estudio.

La supervisión y la evaluación del estudiante bolsista quedan bajo responsabilidad del Centro de Estudios y Perfeccionamiento de cada unidad, donde será controlada frecuencia del mismo y encaminada para Superintendencia de Desarrollo de Recursos Humanos. Sin embargo, cabe a esta la coordinación de estadios.

Las actividades desarrolladas por el estudiante también son evaluadas por el Centro de Estudios y Perfeccionamiento con participación de la Institución de Enseñanza. Al final del estadio el estudiante deberá entregar monografía o estudio de caso como trabajo de conclusión de estadio, que será encaminado para la Superintendencia de Gestión Participativa y Educación en Salud. 
Fueron entrevistados 19 estadiarios de tres áreas del conocimiento en Salud: Enfermería, Farmacia y Medicina. En Enfermería fueron encontrados siete (7) estudiantes y un (1) estudiante del curso técnico. En Farmacia solo un (1) estadiario y en Medicina, diez (10) estudiantes, como muestra el Gráfico 1.

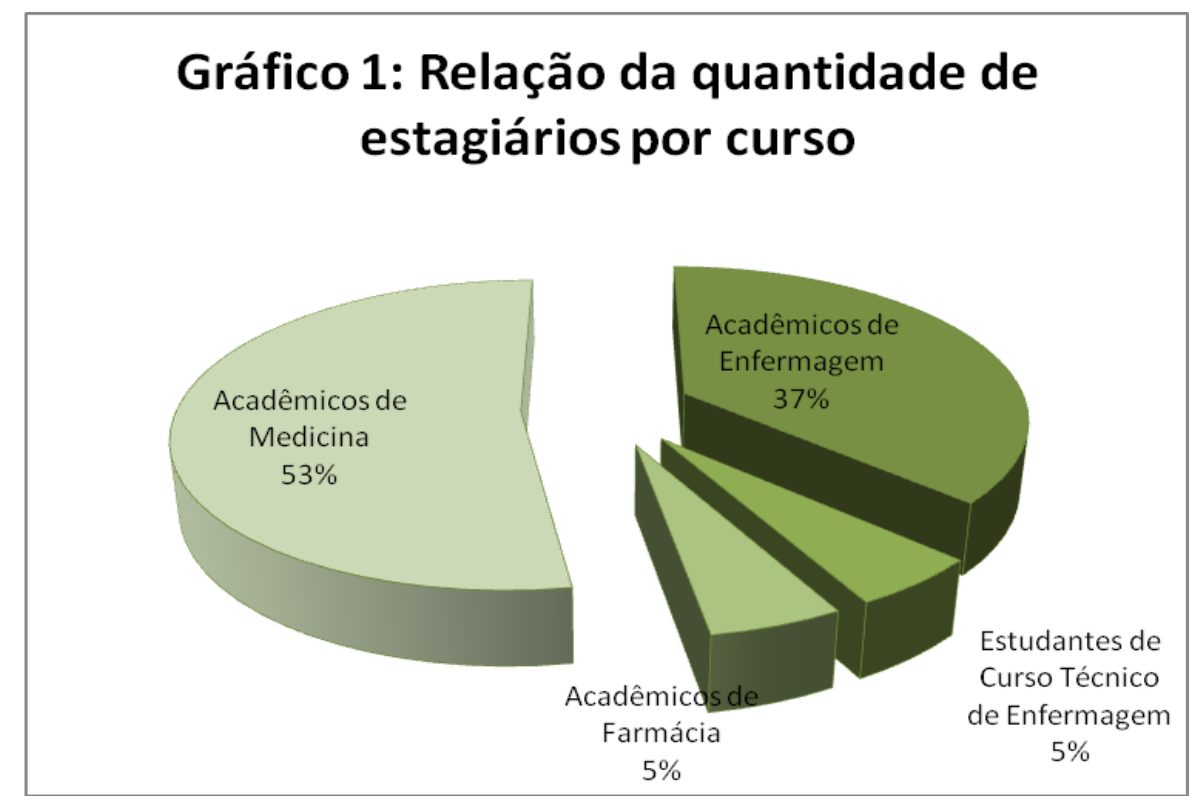

Rio de Janeiro,2008

Los estadiarios fueron entrevistados de acuerdo con su distribuciones en las seis unidades de Salud vinculadas a la SESDEC/RJ, como explicitado en el Cuadro 1.

Cuadro 1. Referente a distribución de los estadiarios en las unidades de Salud vinculadas a la SESDEC/RJ

\begin{tabular}{|ccccc|}
\hline UNIDAD/ & ENFERMERÍA & TÉCNICO EN & MEDICINA & FARMACIA \\
CURSO & 4 & - & 2 & - \\
HEPII & 1 & 1 & 3 & - \\
HEAPN & 1 & - & 2 & - \\
HEAS & 1 & - & 1 & - \\
HERF & - & - & 2 & - \\
HEGV & - & - & - & 1 \\
HEMORIO & & &
\end{tabular}

Rio de Janeiro, 2008

\section{2) LA EVALUACIÓN DEL ESTADIO EXTRACURRICULAR}

Los Centros de estudios y perfeccionamiento (CEA) de cada unidad trazan estrategias evaluativas tanto del conocimiento del estadiario como de las condiciones operacionales de los escenarios de actuación.

De acuerdo con los estadiarios los métodos evaluativos varían teniendo en consideración el curso y la disponibilidad del jefe de equipo o del preceptor. Algunos estudiantes son evaluados a través de la presentación de estudios de casos, reportes de pesquisa, registro 
de asistencia prestada, participación en las reuniones, elaboración de trabajo de conclusión de estadio, registro en los libros de órdenes y ocurrencias donde son colocadas las actividades realizadas.

Considerando las Directrices Curriculares y la necesidad de integración estudio trabajo propuesta con la Legislación de estadio, percibimos como necesaria la distribución de conocimientos teórico prácticos que atiendan las competencias y habilidades generales y que acompañen la evaluación en los siguientes ámbitos: atención a la salud, tomada de decisiones, comunicación, liderazgo, administración y gerencia y educación permanente ${ }^{16}$.

Hubo la información de un estudiante de Medicina que no existe la modalidad formal de evaluación:

"Recibí un impreso (del CEA) en este momento. No voy a poder elaborar lo que la evaluación solicita".

Esta información de evaluación ocurrió cuando la unidad de salud recibió a los investigadores para la colecta de datos.

En algunas unidades, hay reuniones diarias, como en el caso del estudiante del curso técnico en Enfermería, que es evaluado por la profesora supervisora al final del estadio de cada sector. Hay evaluaciones semanales, mensuales y hasta incluso semestrales o, en otros casos, no hay evaluación formal. La práctica de la autoevaluación es puntual y, normalmente, es hecha a través de conversaciones informales con los propios colegas de estadio, algunas personas del sector de estadio o con el CEA.

Los estadiarios luchan con facilidades y enfrentamientos junto al contexto operacional, que es parte del sistema modulado por la Gestión participativa y educación en salud de la SESDEC/RJ en acción junto a las unidades de Salud vinculadas al mismo órgano.

Para algunos estudiantes, como el académico de Farmacia, no hay problemas en el cotidiano del estadio. Esta afirmación se opone a las opiniones de la gran mayoría de los estadiarios, ya que hay quejas sobre la falta de materiales, lechos, literas y medicaciones, precariedad de aparejos, ausencia de preceptores, desinterés de los equipos por los estadiarios y, de acuerdo con un estudiante de Enfermería: "Quiebra de la salud pública".

\section{CONSIDERACIONES FINALES}

Estadio es un proceso didáctico-pedagógico que posibilita al educando contacto con una actividad real, para adquisición de experiencias ${ }^{1}$.Los estadios extracurriculares en Salud, son, ante todo, oportunidades de crecimiento y maduración de la profesión escogida, alternativa para legitimar el carácter ético del profesional de Salud. Y un proceso formador y puntuado en vivencias que otorgan al estadiario cierto grado de madurez.

La ausencia de evaluaciones en algunas ulnidades de Salud confrontan el proceso de aprendizaje, ya que, evaluar, forma parte del aprendizaje. En esos casos, surgen indagaciones sobre lo que la vivencia de estadio ayudó en la formación del estudiante o aún, si un profesional malformado reflejará esa realidad incoherente de su campo de estadio en el futuro.

La disparidad operacional de ciertas unidades de Salud forma el escenario ideal para la inseguridad, ansiedad y error por parte del estadiario. En el proceso de aprendizaje, es 
necesario conocerse el correcto, el patrón. Sin embargo, como declaran los estudiantes, la práctica queda dificultada, atrasada, ante tantos enfrentamientos. La falta de apoyo y las necesidades prioritarias de los estudiantes son tan opuestos en esos contextos, que la motivación al estadio disminuye, a punto de igualarse apenas a la motivación dada por la bolsa auxilio.

Integrar los conceptos de cualificación de los estadiarios a la realidad de que ellos aún se encuentran en un continuo proceso de aprendizaje son puntos importantes a ser considerados por los núcleos que fomentan, regularizan y evalúan las actividades de estadio extracurricular en Salud. Ofrecer el escenario ideal a la práctica y al crecimiento, proporcionará al profesional seguridad e independencia, posibilitándolo a superar las más diversas dificultades, teniendo en cuenta que estará prevenido de estrategias de enfrentamiento.

\section{REFERÉNCIAS}

1. Santos MSS. A Construção de um Projeto Integrado para a Formação do

Enfermeiro:Aliança Estágio Curricular e Extracurricular [tese]. Ribeirão Preto (SP): Escola de Enfermagem de Ribeirão Preto, Universidade de São Paulo; 2005.

2. Secaf V; Lorencette DAC; Marx LC. Enfermagem:o estágio extracurricular remunerado. 1989. Rev Acta Paulista de Enferm., São Paulo. 2(3):79-85.

3. Pucci B et al. Teoria Crítica e Educação: a questão da formação cultural na Escola de Frankfurt. Petrópolis, Rio de Janeiro: Vozes; São Carlos: EDUFISCAR, p.146;1994.

4. Caldeira VP. A gênese do estágio extracurricular em Enfermagem.Enfermagem 1992. Rev Cad. de Enferm., Belo Horizonte, 1(2):58,out.1992.

5. Caldeira VP. Estágio Extra-curricular: Opção ou Obrigação? Uma Contradição a Ser Superada [dissertação] Rio de Janeiro (RJ): Escola de Enfermagem da Universidade do Rio de Janeiro. Universidade do Estado do Rio de Janeiro, 1992.

6. Cunha LA. A Universidade crítica - O ensino superior na república populista. 2ed. Rio de Janeiro: Francisco Alves, 1989. 267 p.

7. Centro de Integração Empresa-Escola - CIEE.Saiba tudo sobre o CIEE - Especial para as instituições de ensino. São Paulo::CIEE, [s.d.] 50p.

8. Brasil. Lei ํㅜ 11.788. Dispõe sobre o estágio de estudantes; altera a redação do art. 428 da Consolidação das Leis do Trabalho - CLT, aprovada pelo Decreto-Lei $n^{\circ} 5.452$, de $1^{\circ}$ de maio de 1943, e a Lei $n^{0}$ 9.394, de 20 de dezembro de 1996; revoga as Leis $n^{\text {os }} 6.494$, de 7 de dezembro de 1977, e 8.859, de 23 de março de 1994, o parágrafo único do art. 82 da Lei $n^{\circ}$ 9.394, de 20 de dezembro de 1996, e o art. $6^{\circ}$ da Medida Provisória $n^{0} 2.164-41$, de 24 de agosto de 2001; e dá outras providências. 25 de setembro de 2008.

9. Rio de Janeiro. Resolução SESDEC n 315 . Secretaria de Estado de Saúde e Defesa Civil. Ato do Secretário. 30 de maio de 2008. Publicado no D.O. no 110 de 19 de junho de 2008.

10. Spangol CA et al.Comissão de Estágios em Enfermagem:A experiência das clínicas da Universidade Federal de Minas Gerais. 2004. Rev Mineira de Enferm, Minas Gerais. 8(2): 326-329.

11. Taquete SR; Macedo LMC; Alvarenga FBF. Comissão de Estágios em Enfermagem: A experiência das clínicas da Universidade Federal de Minas Gerais. 2004. Revista Mineira de Enfermagem, Minas Gerais. 8(2): 326-329.

12. Wolfovitch C; Farias FC; Silva, GCX; Fernandes JD; Souza MG. Experiência de campo necessária à formação do(a) enfermeiro(a). 1975. Rev. Bras. de Enferm., Rio de Janeiro.28(1): 26-55. 
13. Rodrigues MSP; Leitão GCM. Estágio curricular supervisionado com ênfase no desenvolvimento da autonomia e da responsabilidade. 2000. Rev Texto Contexto de Enferm., Florianópolis. 9(1): 216-229.

14. Richardson RJ et al. Pesquisa Social: métodos e técnicas. 3.ed. São Paulo: Atlas, 1999. 15. Triviños ANS. Introdução à pesquisa em ciências sociais: a pesquisa qualitativa em educação. São Paulo:Atlas.1987.

16. Brasil. Ministério da Educação e do Desporto. Conselho Nacional de Educação. Câmara de Educação Superior. Institui Diretrizes Curriculares Nacionais do Curso de Graduação em Enfermagem. Resolução CNE/CES no. 3/2001. Diário Oficial da União, Brasília, Seção 1, p. 37, 09 novembro 2001. 